\title{
Corrigendum to "Bioinformatics Analysis Reveals the Altered Gene Expression of Patients with Postmenopausal Osteoporosis Using Liuweidihuang Pills Treatment"
}

\author{
Rui Gong, ${ }^{1,2,3}$ Shan Ren, ${ }^{4}$ Menghui Chen, ${ }^{5}$ Yanli Wang, ${ }^{6}$ Guoliang Zhang, ${ }^{5}$ Lijuan Shi, \\ Cuizhao Zhang, ${ }^{8}$ Ruihong Su, ${ }^{8}$ and Yukun Li ${ }^{3}{ }^{3}$ \\ ${ }^{1}$ Hebei Medical University Endocrine Teaching and Research Section, China \\ ${ }^{2}$ Department of Gerontology, The Third Hospital of Shijiazhuang, China \\ ${ }^{3}$ Department of Endocrinology, Third Affiliated Hospital of Hebei Medical University, China \\ ${ }^{4}$ Department of ICU, Hebei General Hospital, China \\ ${ }^{5}$ Department of Cardiothoracic Surgery, The Third Hospital of Shijiazhuang, China \\ ${ }^{6}$ Obstetrics and Gynecology, The Third Hospital of Shijiazhuang, China \\ ${ }^{7}$ Department of Endocrinology, The Third Hospital of Shijiazhuang, China \\ ${ }^{8}$ Department of Laboratory, The Third Hospital of Shijiazhuang, China \\ Correspondence should be addressed to Yukun Li; yukunli1225@126.com
}

Received 2 July 2019; Accepted 4 July 2019; Published 20 November 2019

Copyright (c) 2019 Rui Gong et al. This is an open access article distributed under the Creative Commons Attribution License, which permits unrestricted use, distribution, and reproduction in any medium, provided the original work is properly cited.

In article titled "Bioinformatics Analysis Reveals the Altered Gene Expression of Patients with Postmenopausal Osteoporosis Using Liuweidihuang Pills Treatment" [1], there was an error in the affiliation details of Rui Gong and Yukun Li as shown below.

Rui Gong was only affiliated to affiliation number 1 "Hebei Medical University Endocrine Research Institute." However, they should be affiliated to affiliations 1, 2, and 3 .

Yukun Li was wrongly affiliated to affiliation number 2 "Department of Gerontology, The Third Hospital of Shijiazhuang, China." However, they should be affiliated to affiliation number 3 "Department of Endocrinology, Third Affiliated Hospital of Hebei Medical University, China."

The corrected authors' list and affiliations are shown above, and it has been added in-line to the published article.

\section{References}

[1] R. Gong, S. Ren, M. Chen et al., "Bioinformatics analysis reveals the altered gene expression of patients with postmenopausal osteoporosis using liuweidihuang pills treatment," BioMed Research International, vol. 2019, Article ID 1907906, 10 pages, 2019. 


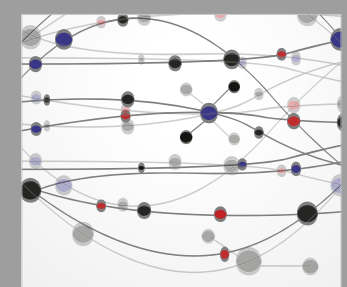

The Scientific World Journal
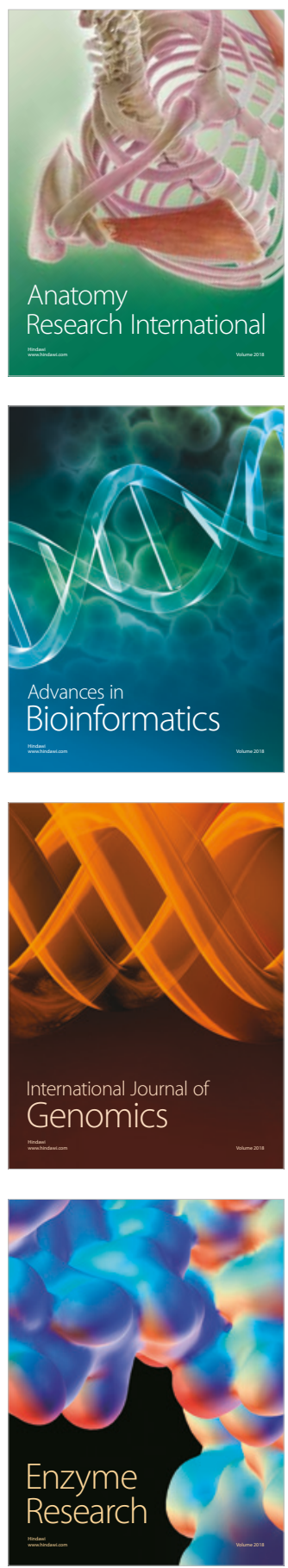
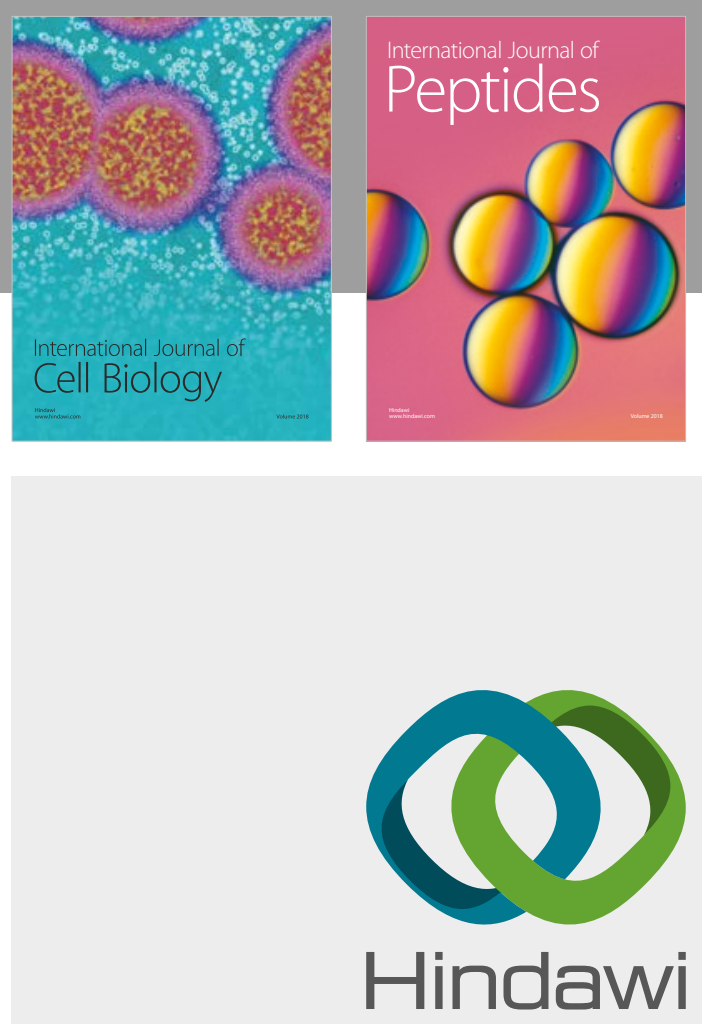

Submit your manuscripts at

www.hindawi.com
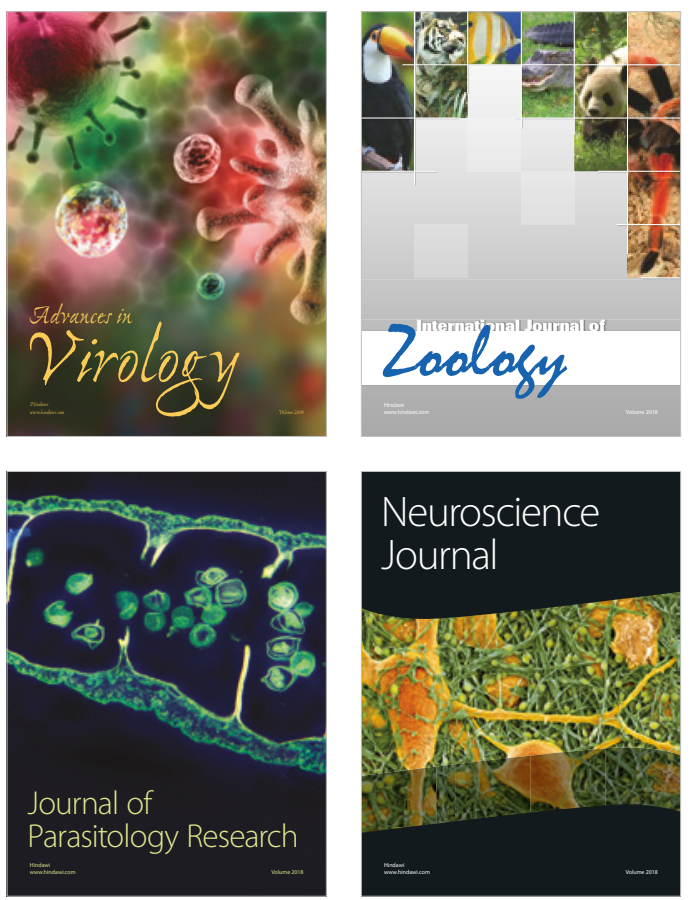
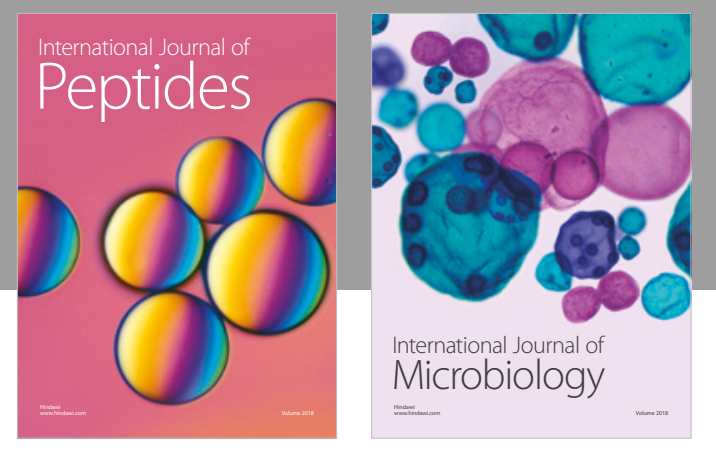

nternational Journal of Microbiology
Journal of
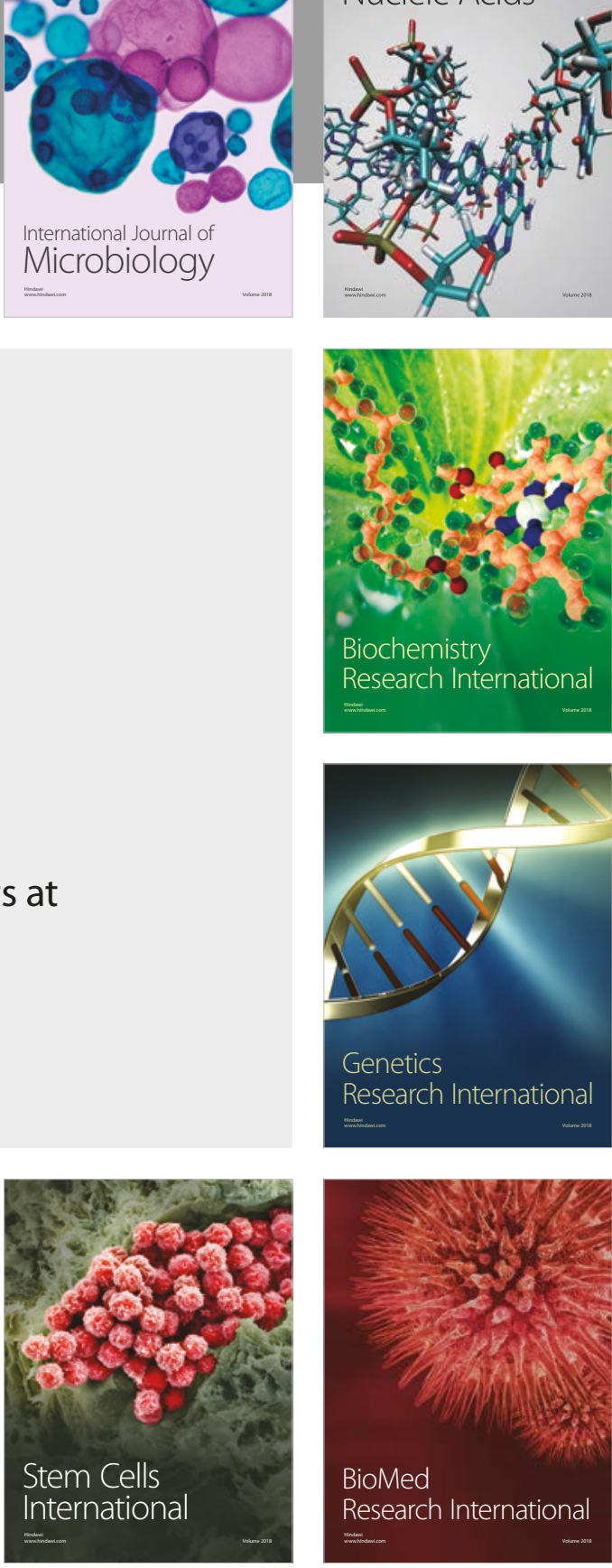
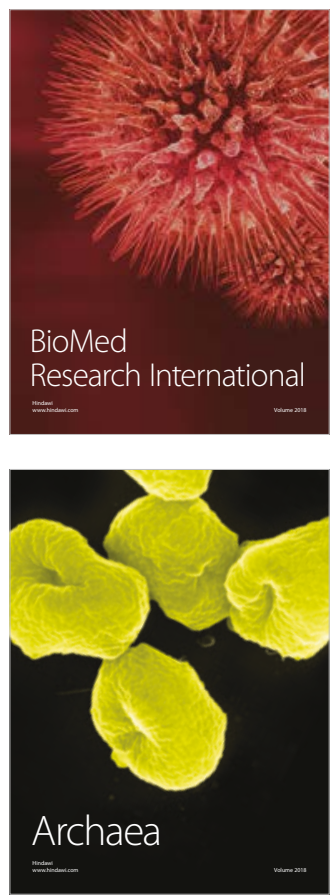